\title{
A Random Dot Stereoacuity Test based on 3D Technology
}

\author{
Angelo Gargantini \\ Università degli Studi di \\ Bergamo \\ Bergamo, Italy \\ angelo.gargantini@unibg.it giancarlo.facoetti@unibg.it
}

\author{
Andrea Vitali \\ Università degli Studi di \\ Bergamo \\ Bergamo, Italy \\ andrea.vitali1@unibg.it
}

\begin{abstract}
This paper presents a novel stereo acuity test that is realized by a software program on a $\mathrm{PC}$ with $3 \mathrm{D}$ capabilities. The $3 \mathrm{D}$ technology is used to provide two separate images to the two eyes. The test is a classical random dot test, but differently to other test printed on paper, the images shown to the patient can vary and there is no monocular clue. These features allow to deliver the test also in groups (instead of individuals) like school classes, and to increase the sensitivity. The system can be easily used also by not specialized personnel and this may further increase the cost efficiency of the test. Experiments carried on with a large set of children of age between five and seven years are presented. The obtained results are discussed through some comparisons between traditional approach and our innovative idea.
\end{abstract}

\section{Keywords}

Stereopsis, Randomdot test, Amblyiopia, 3D

\section{INTRODUCTION}

Amblyopia, otherwise known as 'lazy eye', is reduced visual acuity that results in poor or indistinct vision in one eye that is otherwise physically normal. It may exist even in the absence of any detectable organic disease. Amblyopia is generally associated with a squint or unequal lenses in the prescription spectacles. This low vision is not correctable (or only partially) by glasses or contact lenses. Amblyopia is caused by media opacity, strabismus, anisometropia, and significant refractive errors, such as high astigmatism, hyperopia, or myopia. This condition affects $2-3 \%$ of the population, which equates to conservatively around 10 million people under the age of 8 years worldwide [9]. Children who are not successfully treated when still young (generally before the age of 7) will become amblyotic adults. As amblyotic adults, they will have a normal life, with no proven losses in terms of education and occupation opportunities [3], except that they are prohibited from some occupations and they

Permission to make digital or hard copies of all or part of this work for personal or classroom use is granted without fee provided that copies are not made or distributed for profit or commercial advantage and that copies bear this notice and the full citation on the first page. To copy otherwise, to republish, to post on servers or to redistribute to lists, requires prior specific permission and/or a fee. REHAB 2014, May 20-23, Oldenburg, Germany Copyright $\odot 2014$ ICST 978-1-63190-011-2 DOI 10.4108/icst.pervasivehealth.2014.255333 are exposed to a higher risk of becoming seriously visually impaired. The best described long term consequence of amblyopia is an increased risk of bilateral blindness, caused most frequently by traumatic eye injury in younger people and age related macular degeneration in older people. The projected lifetime risk of vision loss for an individual with amblyopia is estimated around at least 1-2\% [6].

For these reasons screening for amblyopia in early childhood is done in many countries to ensure that affected children are detected and treated within the critical period, and achieve a level of vision in their amblyopic eye that would be useful should they lose vision in their non-amblyopic eye later in life. However, there is no strong scientific evidence that preschool vision screening is cost-effective and some researchers suggest that health authorities should stop purchasing pre-school vision screening services [7].

One factor that weakens the effectiveness of amblyopia screening is that population-based screen tests can suffer from a very low sensitivity and specificity. Several methods are available for the measurement of stereopsis. The most common procedures are anaglyph (with use of red-green filters) and vectographic (with use of polarized filters) procedures. However, these tests since they have monocular clues [5] and may result difficult to pass even for normal people can have low sensitivity and specificity. In one proven case, screening for amblyopia by the local nurses using the visual acuity tests or random dot stereopsis test alone displayed a very low sensitivity (around 20\%) [2].

In this paper we present our research project that has the main goal of developing a method (called 3DSAT) for stereopsis test that should not suffer from the typical weaknesses of classical stereo tests. 3DSAT aims to be:

- Very easy to deliver at a low cost. Even unqualified personnel can perform the test, thus increasing the widespread use and reducing the cost of the test.

- With a very high sensitivity: its measure of the proportion of actual amblyopic positives which are correctly identified as such is equal to $100 \%$. In this way it reduces the risk of undetected amblyopia.

- With an acceptable sensitivity: its measure of the proportion of negatives which are correctly identified as such is near to $100 \%$. This has the goal of avoiding frequent visits with more qualified (and costly) personnel. 


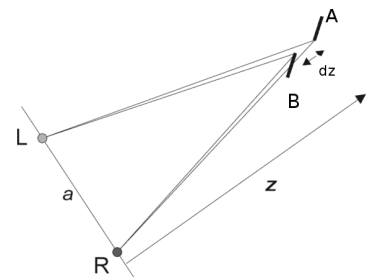

Figure 1: Mathematical model for stereo acuity.

3DSAT exploits a 3D computer system, a modern technology able to reproduce stereo images on computer screens. There are already some works on using 3D monitor for stereotest and for amblyopia treatment [8]. Our method advances w.r.t. [4] because it implements Random dot instead of Wirt tests, and extends the work presented in [1] with more advances features (like use of active $3 \mathrm{D}$ glasses, use of multiple images instead of simple random dot circles).

In this paper, first we describe stereo acuity and its evaluation using random dot test through the development of an application exploiting the 3D technology. Then, we show the test procedure defined to execute the tests on a set of children using this application. Finally, we show and analyze the results of this experiment by highlighting pros and cons of our research work.

\section{3DSAT: A 3D STEREO ACUITY TEST} 2.1 Stereoscopic acuity and its measurement

Having two eyes, as being humans and most animals, located at different lateral positions on the head allows binocular vision. It allows for two slightly different images to be created that provide a means of depth perception. Through highlevel cognitive processing, the human brain uses binocular vision cues, such as binocular disparity, discrimination of object size, and surface orientation to determine depth in the visual scene. This particular brain skill is defined as stereopsis. Some pathologies are caused by total or partial stereopsis absence, such as blindness on one eye and strabismus. The examination of stereopsis ability can be evaluated by measuring stereoscopic acuity.

Stereoscopic acuity, also named stereoacuity, is the smallest detectable depth difference that can be seen by someone with two eyes and normal brain functions. It had been invented by Howard and Dolman who explained stereoacuity with a mathematical model as follows in figure 1. The observer is shown a black peg at a distance of $6 \mathrm{~m}(=20$ feet $)$. A second peg, below it, can be moved back and forth until it is just detectably nearer than the fixed one. Stereoacuity is this difference in the two positions, converted into an angle of binocular disparity. Equation 1 shows how to convert $\delta \mathrm{z}$ in order to perform the angle of disparity $\delta \mathrm{y}$ in arc of minutes.

$$
\delta y=c a \frac{\delta z}{z^{2}}
$$

In equation 1, $a$ is the interocular separation of the observer and $z$ the distance of the fixed peg from the eye. To transfer $\delta \mathrm{y}$ into the usual unit of minutes of arc, a multiplicative constant $c$ is inserted whose value is 3437.75 ( 1 radian in arcminutes).

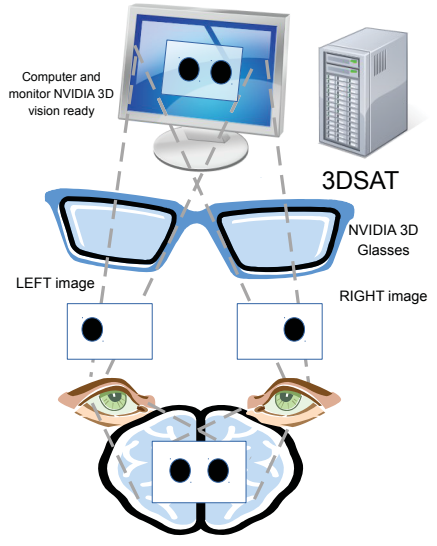

Figure 2: Using 3D to separate the dots

At present, there are different procedures to measure stereoacuty without using cumbersome two-peg device. These tests can measure stereacuity using either real stereogram or the emulation of stereogram using 3D technology. Among these, the Random-dot stereogram (RDS) is widely used because it permits to obtain a test procedure, named Randot stereotest, that is easily administrated and not subject to deception. RDS is stereo pair of images of random dots which when viewed with the aid of a stereoscope, or with the eyes focused on a point in front of or behind the images, produces a sensation of depth, with objects appearing to be in front of or behind the display level. Randot stereotest has been easily emulated using 3D technology in this research work, i.e. a 3D based software, named 3DSAT, has been developed by exploiting IT potentiality. In particular, the random small pattern elements are the pixels of PC screen forming digital images, one behind the other, showed to the patient using 3D glasses. Therefore, formula 1 has been adapted by substituting pegs with pixels and the geometrical models has been redefined according to the use of PC.

\subsection{Using 3D for stereo acuity test}

3DSAT is a system based on a 3D computer system. The current version is based on the NVIDIA $\left(\right.$ B 3 Vision ${ }^{\text {TM }}$ technology, although other 3D technologies may be supported as well in the future. The NVIDIA 3D Vision technology is one of the most accessible 3D technologies available on the market today, it requires a standard personal computer with a NVIDIA graphic card, a monitor 3D Vision ready, which is capable of a refresh rate of $120 \mathrm{Mhz}$, and a NVIDA LCD shutter glasses. The NVIDIA 3D vision is based on active shutter technology. With this method, the left and right eye images are presented on alternating frames, but since these monitors are capable of $120 \mathrm{~Hz}$, each eye still sees a full $60 \mathrm{~Hz}$ signal that is equivalent to the refresh rate on LCD monitors today. This offers a number of advantages with respect to other stereoscopic technologies like polarized or anaglyphs glasses or head-mounted displays, including: full image quality per eye, wide viewing angle for 3D, personalized fit, and acceptable cost.

The system we have developed for 3DSAT consists in a normal PC desktop connected to a 3D monitor (3D VisionReady Display). The PC must be 3D capable and have all 


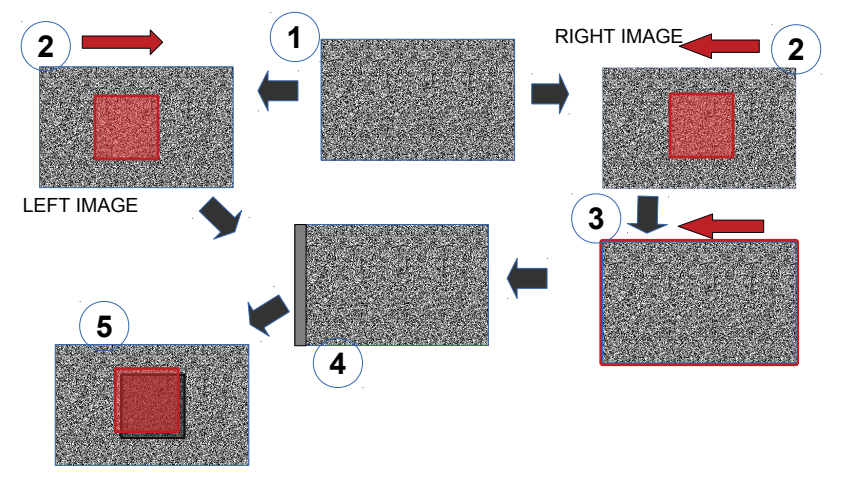

Figure 3: How the 3D image is created

the 3DSAT software installed on it. The patient wears the NVIDIA active LCD shutter glasses that allow viewing a different image from the left and right eye. The scenario is depicted in Figure 2. This principle is the separation of the images provided to the right and left eye.

The stereo image to show to the child is built by the following steps as depicted in Fig. 3.

1. A random dot image is created (every time with different distribution of the dots)

2. The pixels corresponding to the shape to be shown (in the Figure 3 a square) are translated with a desired disparity (in terms of pixels) in two opposite directions for the left and the right image.

3. One of the two images is entirely translated in the same direction.

4. The two images are composed in an unique 3D image shown to the child.

5. The child, in the presence of stereopsis and wearing the 3D glasses, perceives the shape in depth.

The shape is seen in depth because the left eye sees one set of dots and the right eye sees a second set of dots. The dots are identical, except for the dots within the square that have been horizontally displaced (nasally in the figure). During stereopsis the two 2D retinal images are fused into a single $3 \mathrm{D}$ percept. Through identifying corresponding points, 'matching primitives' in the slightly offset retinal images of the left and right eye, the images can be aligned in such a way as to reveal depth information. The nasal displacement produces the stereoscopic perception that the square of circles is raised off the page [10].

The translation of one entire image (step 3) eliminates any clue of the shape one could have without the 3D glasses: if the two images are composed in the same position all the pixels except those translated are in the identical position and are completely overlapped. This would cause an increase in the composed image of the density of the pixels in correspondence of the shape. Wearing the glasses would eliminate the diversity of density but without the glasses one would have a clue of the shape. By translating one image we restore an equal density of the dots.

The 3DSAT software has been implemented using Java and

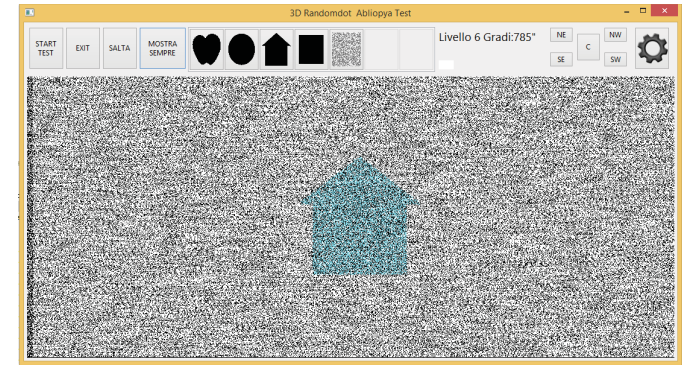

Figure 4: User interface of 3dsat application

\begin{tabular}{cccr} 
Dim. & Resolution & Distance & Minimum angle \\
\hline $23 "$ & FHD 1920 x 1080 & $40 \mathrm{~cm}$ & $136.8 "$ \\
$23 "$ & FHD 1920 x 1080 & $80 \mathrm{~cm}$ & $68.4 "$ \\
$27 "$ & FHD 1920 x 1080 & $40 \mathrm{~cm}$ & $160.5 "$ \\
$27 "$ & FHD 1920 x 1080 & $120 \mathrm{~cm}$ & $53.5 "$
\end{tabular}

Table 1: Minimum angle definition according to dimensions, resolution and distance of the PC screen.

OpenGL and works under Microsoft Windows systems. A screenshot of the software is shown in Fig. 4. At the beginning the user inserts the patient data, selects an image set $^{1}$, the starting disparity, the distance of the child from the screen, the dimension of the screen and the dimension of the shapes. After a preview of what the shapes will look like (as shown in Fig. 4), the software randomly chooses a shape in the shape set and shows the 3D random dot stereotest for that shape. The child either says or indicates what it sees and if the guess is right then the software chooses another shape and decreases the angle of disparity. The software assesses a certain level of disparity if the child can guess the shapes for that level for three times with maximum one error. The minimum angle of measurement depends on the monitor and on the distance of the child, and some examples are reported in Table 1.

Policies. In order to reduce the number of false negative (i.e. amblyopic not correctly identified and falsely passing the test) and increase the test sensitivity we have devised the following policies: (i) the shape is randomly chosen every time, (ii) also the control null image (no shape is shown) can be inserted, (iii) either the user that delivers the test has no clue about which shape is currently displayed, (iv) we have checked the absence of any monocular clue by using the software both without glasses and with glasses but with one occluded eye. In both cases we were unable to identify the images.

In order to reduce the number of false positives (i.e. non amblyopic falsely failing the test) and increase the test specificity, we have devised the following policies: (i) the shape is shown as image: if the child has difficulties to recognize the shape and identify its name, he/she can simply point his/her finger, (ii) the test has initial phase in which no measurement is taken and the images are shown colored: in

${ }^{1}$ 3DSAT now provides set of images similar to those presented in the LANG stereotest I and II, TNO, LEA, pacman and letters. New image sets can be easily added. 


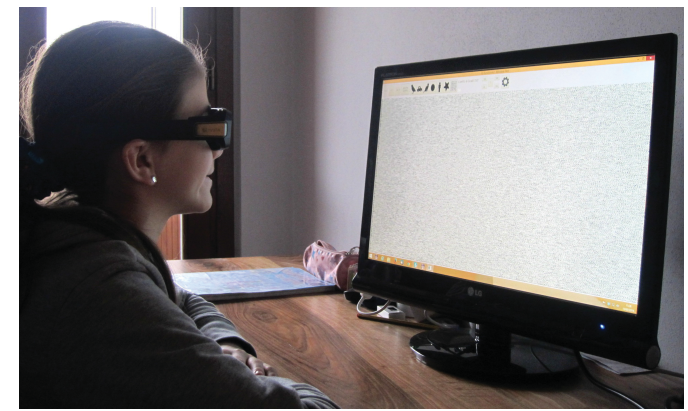

Figure 5: A child is using 3dsat with LCD shutter glasses.

\begin{tabular}{cccc} 
Distance & Mean & Min & Max \\
\hline $40 \mathrm{~cm}$ & $398.12 "$ & $139.12 "$ & $962.02 "$ \\
$50 \mathrm{~cm}$ & $407.44 "$ & $128.27 "$ & $769.62 "$ \\
$60 \mathrm{~cm}$ & $278.84 "$ & $106.89 "$ & $641.35 "$ \\
$80 \mathrm{~cm}$ & $170.41 "$ & $79.49 "$ & $481.01 "$ \\
$100 \mathrm{~cm}$ & $231.12 "$ & $64.13 "$ & $384.81 "$ \\
$120 \mathrm{~cm}$ & $109.32 "$ & $53.44 "$ & $213.78 "$ \\
$150 \mathrm{~cm}$ & $101.06 "$ & $42.75 "$ & $299.29 "$ \\
$160 \mathrm{~cm}$ & $100.21 "$ & $40.08 "$ & $240.50 "$ \\
$200 \mathrm{~cm}$ & $51.30 "$ & $25.65 "$ & $64.13 "$
\end{tabular}

Table 2: Distance from the screen, mean, minimum and maximun stereo acuity values.

this way the child understands what the shape will look like when the actual measurement is started and the color disappears, (iii) when a child fails the test, the test can be retried with a different set of images (after the equipment is checked for failures). Since the images are randomly chosen, the test can be repeated without loss of sensitivity (differently from the classical test on paper).

\section{EXPERIMENT}

We performed some tests with the main aim of verifying if our system does not identify false positives or false negatives. The tests were carried out on a population with age between 5 years and 7 years, with a small presence of amblyopic subjects (known before starting the tests). Due the young age of the people taking the tests, we decided to carry out the tests as a group game. The aim of the game was to guess the shapes visualized on the screen. Obviously, only the child with the $3 \mathrm{D}$ glasses would be able to see the shapes, so children were asked to guess the shapes one after the other. The traditional Lang test would not fit well with this modality, because the young patients would suggest each other the correct results, causing false negatives. Also, our computer-based test permits to have a great number of different shapes, reducing further the number of false negatives (children could take a wild guess without actually see the right given shape) The mean time required to test one person is 2 minutes, faster than the traditional way. In total we tested 90 subjects for a total of 180 test results. We identified 8 potential positive cases, confirmed by teachers. We clustered test data grouping the distance from the screen and the data is shown in Table 2.

Observing the collected data, we can say that the minimum and maximum stereo acuity values decrease linearly with the increasing of the distance. The mean values depend on the stereo acuity of the various subjects taking the tests, however they present too a decreasing trend with the distance increase. This is expected since by increasing the distance, the minimum measurable angle of stereopsis decreases (see Tab. 1)

\section{CONCLUSIONS}

We have presented an application that emulates randomdot test using 3D technology. 3DSAT has been used for our experiment on young children and early results have been considered promising. The test procedure has demonstrated the potentialities of our application compared to the traditional approach. Therefore, our research work proposes an innovative and better approach for this test, which is also frequently used for detecting amblyopia, strabismus and suppression, and to measure stereoacuity.

\section{REFERENCES}

[1] A. Breyer, X. Jiang, A. Rütsche, and D. S. Mojon. A new 3D Monitor-Based random-dot stereotest for children. Investigative ophthalmology $\&$ visual science, 47(11):4842, 2006.

[2] C.-H. Chang, R.-K. Tsai, and M.-M. Sheu. Screening amblyopia of preschool children with uncorrected vision and stereopsis tests in eastern taiwan. Eye, 21(12):1482-1488, Sept. 2006.

[3] B. Chua. Consequences of amblyopia on education, occupation, and long term vision loss. British Journal of Ophthalmology, 88(9):1119-1121, Sept. 2004.

[4] J. L. de Bougrenet de la Tocnaye, B. Cochener, S. Ferragut, D. Iorgovan, Y. Fattakhova, and M. Lamard. Supervised stereo visual acuity tests implemented on 3D TV monitors. Journal of Display Technology, 8(8):472-478, Aug. 2012.

[5] E. Hahn, D. Comstock, S. Connick, J. MacCarron, S. Mulla, P. Peters, and R. LaRoche. Monocular clues in seven stereoacuity tests. Dalhousie Medical Journal, 37(1), 2010.

[6] J. S. Rahi, S. Logan, C. Timms, I. Russell-Eggitt, and D. Taylor. Risk, causes, and outcomes of visual impairment after loss of vision in the non-amblyopic eye: a population-based study. The Lancet, 360(9333):597-602, Aug. 2002.

[7] S. Stewart-Brown and S. Snowdon. Evidence-based dilemmas in pre-school vision screening. Archives of Disease in Childhood, 78(5):406-407, 1998.

[8] A. Vitali, G. Facoetti, and A. Gargantini. An environment for contrast-based treatment of amblyopia using 3D technology. In International Conference on Virtual Rehabilitation 2013 - August 26-29, 2013 in Philadelphia, PA, U.S.A., 2013.

[9] A. L. Webber and J. Wood. Amblyopia: prevalence, natural history, functional effects and treatment. Clinical and Experimental Optometry, 88(6):365-375, Nov. 2005.

[10] K. Wright. Handbook of pediatric strabismus and amblyopia. Springer, New York ;;London, 2006. 